\title{
Contrast Enhancement Patterns of Non-Traumatic Brain Lesions: A Diagnostic Approach on Magnetic Resonance Imaging
}

\author{
Chetan M Mehta ${ }^{1}$, Ayaz I Dabivala ${ }^{2}$ \\ ${ }^{1}$ Professor and Head, Department of Radiology, ${ }^{2}$ Senior Resident, Department of Radiology, Medical College and Sir Sayajirao \\ Hospital, Maharaja Sayajirao University, Vadodara-390001, Gujarat, India
}

Corresponding author: Dr. Ayaz I Dabivala, 203, Lakshya Complex, Next To Hotel Subaelite, Fatehgunj Main Road, Vadodara-390002, India

DOI: http://dx.doi.org/10.21276/ijcmsr.2020.5.1.39

(cC) BY-NC-ND

How to cite this article: Chetan M Mehta, Ayaz I Dabivala. Contrast enhancement patterns of non-traumatic brain lesions: a diagnostic approach on magnetic resonance imaging. International Journal of Contemporary Medicine Surgery and Radiology. 2020;5(1):A173-A178.

\section{A B S T R A C T}

Introduction: Contrast enhanced imaging for focussing a differential diagnosis is well known in central nervous system (CNS) imaging. Specific patterns of contrast enhancement on Magnetic resonance imaging (MRI) can be used as a signature to diagnose many conditions with great confidence. This study highlights the use of patterns of contrast material enhancement in non traumatic brain lesions on MRI Brain, to define the locations and appearances of the lesions with specific patterns and narrow the differential diagnosis to a specific etiology.

Method and Materials: This was a record based cross sectional study of 113 cases referred for contrast enhanced MRI of brain done from September 2017 to august 2018. Any patient whose MRI Brain showed contrast enhancing lesion(s) and/ or detected incidentally was included without any exclusion to specific age or gender. Any patient with enhancing lesions in brain secondary to alleged history of trauma were excluded. All the data collected were compiled as per patient and contrast enhancement pattern in Microsoft excel and descriptive statistics in the form of percentage,and mean were represented in the form of tables and charts.

Results: Of the 133 instances of enhancement patterns detected: 56.63\% (64) of the enhancement patterns were infective etiology, most common being smooth thin ring enhancement(25.56\%, 34 cases); $36.28 \%$ (41) neoplasms of which pachymeningeal enhancement(14.28\%) was most common.

Conclusions: Specific enhancement patterns of different etiologies enable non-invasive diagnosis of neurological lesions and improve patient care by instituting prompt treatment and minimizing the unnecessary investigations.

Keywords: Brain, Contrast, Central Nervous System, Enhancement, Patterns, Magnetic Resonance Imaging

\section{INTRODUCTION}

Central nervous system(CNS) lesions are commonly encountered in day to day practice and result in significant morbidity and mortality. ${ }^{1,2,3}$ Excellent soft tissue contrast, ability to delineate brain parenchyma and cranial nerves and non invasive ionizing radiation free imaging makes Magnetic resonance imaging (MRI) the modality of choice for the diagnosis of CNS disorders. ${ }^{3,4} \mathrm{MRI}$ of brain aims to establish a diagnosis and differential diagnosis, characterise the lesions, delineate the anatomic relations of lesion with respect to gray and white matter for therapeutic planning and interventions. ${ }^{5}$ Contrast enhanced MRI improves image contrast increasing lesion conspicuity as well as facilitating diagnosis, detection, follow up and treatment. ${ }^{4}$ Different pathologic processes result in abnormal contrast enhancement using gadolinium based contrast enhanced MRI.

Alterations of blood brain barrier due to angiogenesis, acute inflammation (infectious and non-infectious), cerebral ischemia and pressure overload (eclampsia, hypertension) result in what is known as interstitial or extravascular enhancement. Examples of interstitial enhancement include leakage of fluid into the interstitium from low grade fluid secreting neoplasms (hemangioblastoma. Ganglioglioma, pleomorphic xanthoastrocytoma) and venous perivascular inflammation in demyelination from multiple sclerosis (MS). Abscess enhancement results from both increased vascularity and abnormal permeability.

Another type of enhancement is known as intravascular enhancement which occurs due to abnormal retention of contrast medium in the brain vessels and is proportional to increases in blood flow. It usually reflects neo-vascularity, vasodilatation-hyperemia and short transit time or shunting. It is commonly seen in infarcted territory during the first week and reflects lepto-meningeal inflammation, encephalitis, or peri-venular compromise of the blood-brainbarrier with alterations in cerebellar blood flow. Knowledge of the patterns and mechanisms of contrast enhancement facilitate a radiologic differential diagnosis., ${ }^{1,2,3}$

This study highlights the use of patterns of contrast material enhancement in non traumatic brain lesions on MRI Brain, 
to define the locations and appearance of the lesions with specific patterns and narrow the differential diagnosis to a specific etiology.

\section{MATERIAL AND METHODS}

This was a record based cross sectional study of 113 cases referred for magnetic resonance imaging study from September 2017 to august 2018 for a period of 12 months in compliance with the guidelines and recommendations of the hospital's Institutional ethics committee for human research(IECHR). Any patient whose MRI Brain showed contrast enhancing lesion(s) and/or detected incidentally was included without any exclusion to specific age or gender. Any patient(s) with enhancing lesions in brain secondary to alleged history of trauma were excluded. MRI was performed on General Electronics(GE) 1.5 TESLA MR Electromagnet. Dedicated head coil, standard surface coils and body coils, were used for brain, cervical, thoracic and lumbar spine for acquisition of images. Conventional MR imaging was performed by taking axial T1-weighted, axial and sagittal T2-weighted, fast fluid attenuated inversion recovery (FLAIR) images in coronal plane. Contrast enhanced MRI was performed in axial T1 weighted fat suppressed planes using gadopentetate dimeglumine (Magnilek,UNIQUE PHARMACEUTICALS, BHARUCH) in a standard dose of $0.1 \mathrm{mmol} / \mathrm{kg}$. Post processing in to post contrast images in coronal and sagittal plane was done.

Only those MRI scans which showed enhancing lesions were analysed. Analyses of MRI brain was performed by routine analyses of extra axial spaces, ventricular system, basal cisterns, Cerebral parenchyma, Midline shift, Cerebellum, and Brain stem. Presence of enhancing lesions was documented in the form of number of lesions, predominant localization, morphology of lesions and intrinsic lesion architecture. Then, the lesions were grouped as per their patterns of contrast enhancement as elucidated by smirniotopoulos et al in 20071: a) Linear pachymeningeal b) Leptomeningeal c) Gyral d) Nodular subcortical e) Ring enhancement (smooth thin or thick irregular or incomplete or open ring or Deep and periventricular) and f) Periventricular enhancement(Smooth and thin or Irregular and thick). Lastly, any pathological cranial nerve enhancement was documented and grouped separately. Any enhancement pattern not following the above classification was grouped as unclassified/indeterminate.

All the data collected were compiled as per patient and contrast enhancement pattern in Microsoft excel and descriptive statistics in the form of percentage, and mean were represented in the form of tables and charts. Data collected was also tabulated and distributed as per the etiology and location of the lesion. Various lesions demonstrating

\begin{tabular}{|c|c|}
\hline Enhancement pattern & Major differential diagnosis \\
\hline 1) Linear Pachymeningeal enhancement & $\begin{array}{l}\text { 1) Post surgical } \\
\text { 2) Spontaneous intracranial hypotension } \\
\text { 3)Secondary CNS lymphoma } \\
\text { 4)Meningioma }\end{array}$ \\
\hline 2) Leptomeningeal (pia-arachnoid) enhancement & $\begin{array}{l}\text { 1) Meningitis } \\
\text { 2) meningoencephalitis }\end{array}$ \\
\hline 3) Superficial gyral enhancement & $\begin{array}{l}\text { 1)After reperfusion in cerebral ischemia } \\
\text { 2)Vasodilatation phase of migraine } \\
\text { 3)Cerebral infarction(Healing phase) } \\
\text { 4)Posterior reversible encephalopathy syndrome } \\
\text { 5)Vasodilatation with seizures } \\
\text { 6)Encephalitis } \\
\text { 7)Meningitis }\end{array}$ \\
\hline 4) Nodular cortical-subcortical enhancement & $\begin{array}{l}\text { 1)Metastasis } \\
\text { 2)Septic emboli }\end{array}$ \\
\hline $\begin{array}{l}\text { 5) Ring enhancement } \\
\text { (A) Smooth, thin }(2-7 \mathrm{~mm}) \text {, complete }\end{array}$ & 1)Organizing abscess \\
\hline (B) Thick, irregular, shaggy margins $(>10 \mathrm{~mm})$ at least in some area & 1)Necrotic neoplasm \\
\hline (C) Incomplete ring/open ring & $\begin{array}{l}\text { 1)Multiple sclerosis(without mass effect) } \\
\text { 2)Tumefactive demyelination(with mass effect) } \\
\text { 3)Fluid secreting neoplasm(with mass effect and surrounding } \\
\text { vasogenic edema) }\end{array}$ \\
\hline (D) Deep and periventricular & $\begin{array}{l}\text { 1)Toxoplasmosis } \\
\text { 2)Primary CNS lymphoma }\end{array}$ \\
\hline $\begin{array}{l}\text { 6) Periventricular enhancement } \\
\text { (A) Thick, irregular(homogenous lamb's wool pattern on CECT) } \\
\text { (B) Thin, smooth }\end{array}$ & $\begin{array}{l}\text { 1)Primary CNS lymphoma } \\
\text { 1)Infectious ependymitis }\end{array}$ \\
\hline
\end{tabular}


patterns of contrast enhancement were grouped accordingly. Lesions were further grouped as per their signal intensities in other sequences and provisional imaging diagnosis. Final confirmatory diagnosis was affirmed as per Clinical / Radiological / Histopathological features.

\section{RESULTS}

A total of 113 cases which showed enhancing lesions on MRI brain were studied. The study population included a total of 45 females and 68 male patients with the youngest patient in our study a 45 days old female and the oldest patient aged $75 y e a r s$. The mean of age parameters was about 32.38 years with a standard deviation of 21.76 years.

Smirniotopoulos et al 2007 have proposed 11 contrast enhancement patterns. The same patterns were used in our study for classification. However, some lesions did not confine to those patterns and hence were grouped as unclassified as of yet and the lesions showing pathological cranial nerve enhancement were grouped separately. Predominant etiological distribution of enhancement patterns is illustrated in Figure 1. Maximum number of cases in our study showed smooth thin ring enhancement, accounting for $25.56 \%$ (34) of the total patterns detected. Leptomeningeal enhancement pattern formed the next bulk of cases detected in our study (21.80\%). 7.5\% (10) other patterns were detected which did not conform to the patterns of enhancement as proposed by smirniotopoulos et al 2007. On elaborating the detailed etiological analyses of enhancement patterns, we found that $56.63 \%$ (64) of the enhancement patterns were seen in infective cases, whereas $36.28 \%$ (41) of the enhancement patterns facilitated the diagnosis of neoplasms. Other minor etiologies were also detected from enhancement patterns in our study: 2 cases of hematological disorder(bone marrow enhancement), 2 cases of developmental malformation(cavernous angiomas), and 1 case each of metabolic, inflammatory and demyelinating(multiple sclerosis) diseases. One case showed contrast enhancement, however no etiological diagnosis was reached clinically or radiologically. The

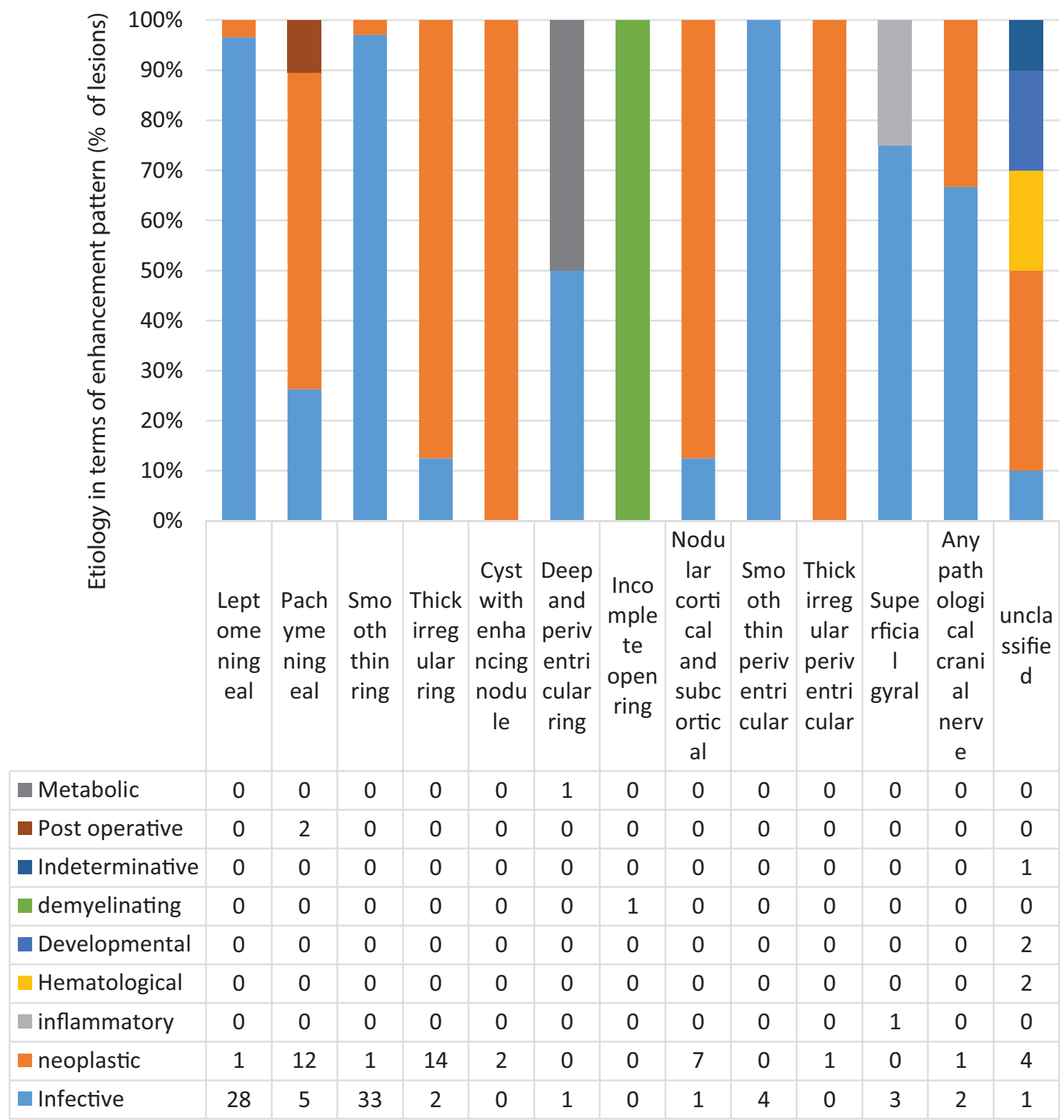

Figure-1: Distribution of patterns of contrast enhancement as per etiology 


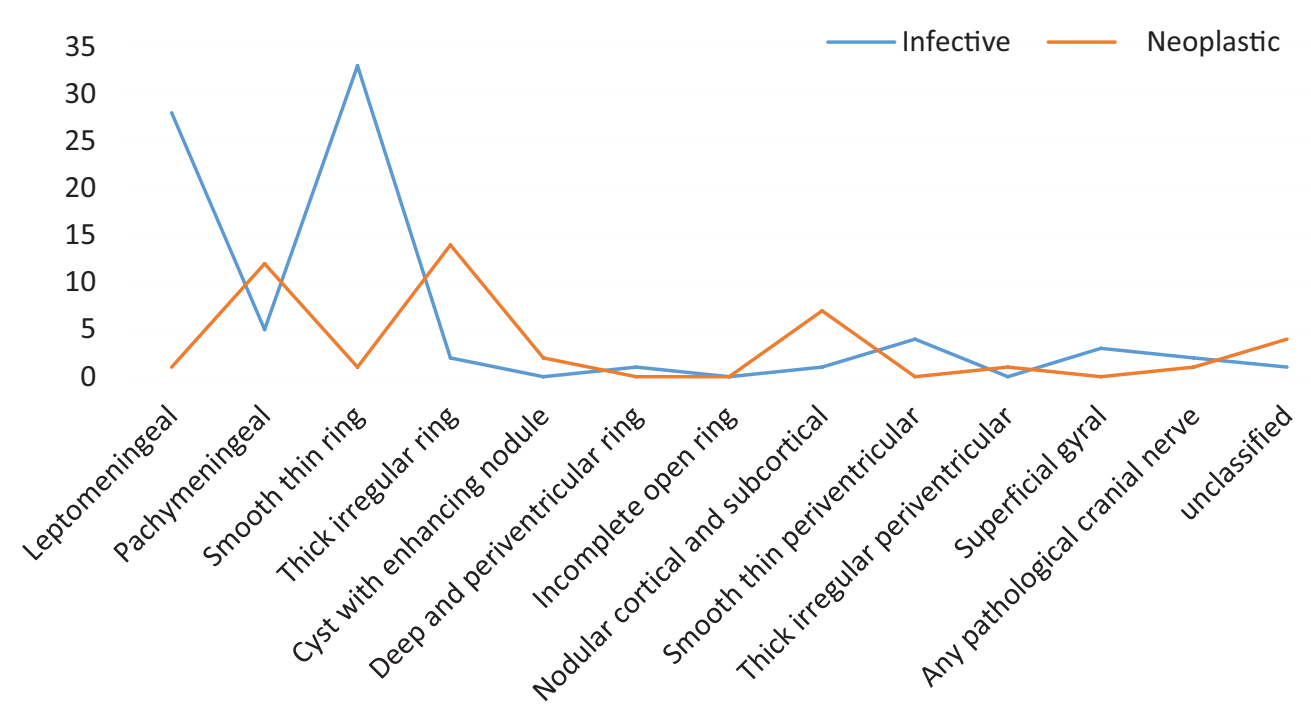

Figure-2: Comparison of enhancement patterns across infective and neoplastic etiology
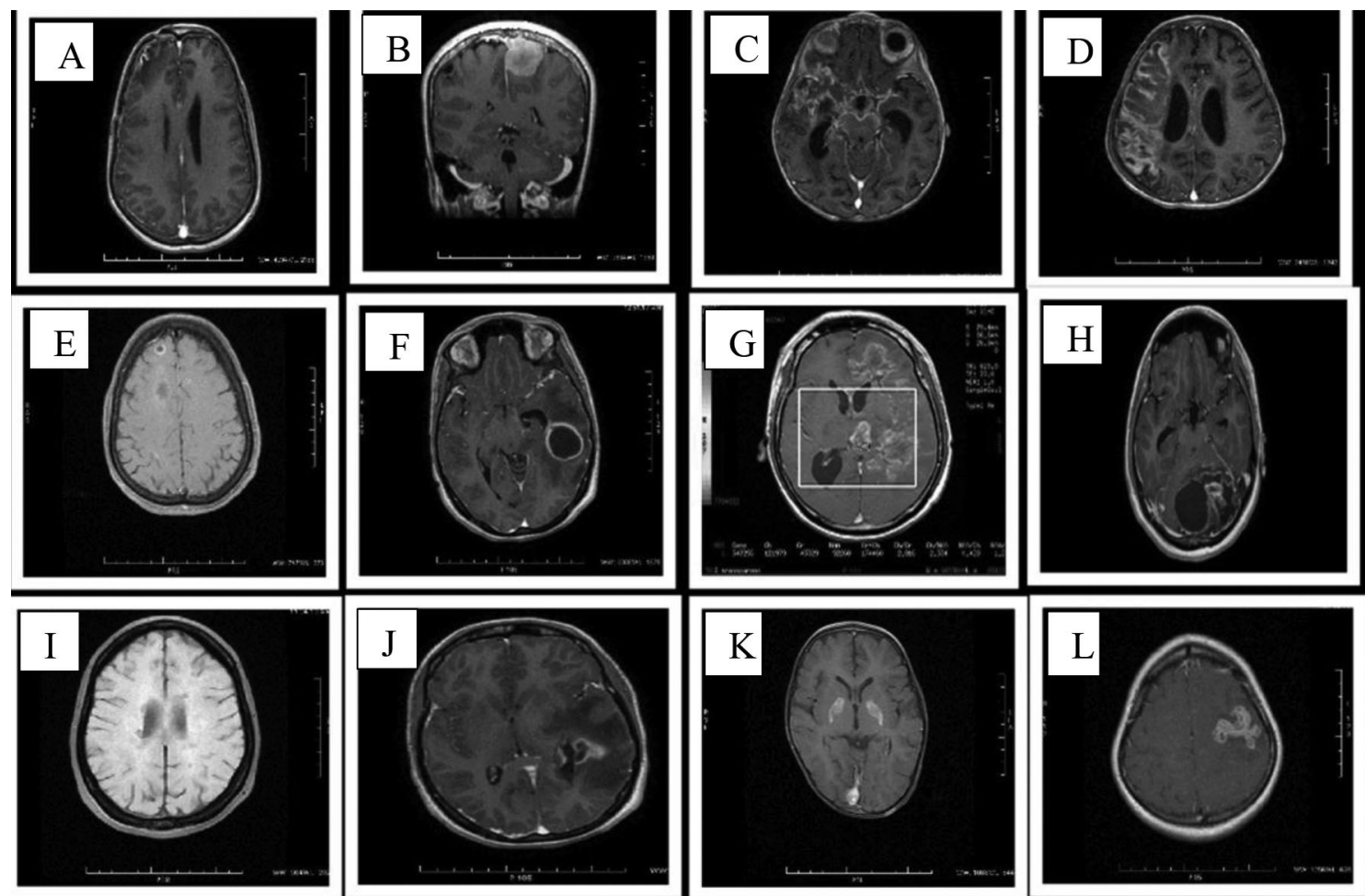

Figure-3: Patterns of contrast enhancement(Etiologies): A) Post operative Pachymeningeal enhancement(iatrogenic) B) Dural tail: Pachymeningeal enhancement of meningioma(neoplasm) C) Leptomeningeal enhancement of Tuberculosis(infective granulomatous) D) Superficial gyral enhancement of viral encephalitis(infective) E)Nodular cortical subcortical enhancement of metastasis(neoplasm) F) Smooth thin ring enhancement of Pyogenic brain abscess(infective) G) Thick irregular ring enhancement of high grade glioma (neoplastic) H) Cyst with enhancing mural nodule in pilocytic astrocytoma(neoplastic) I) Incomplete ring enhancement of multiple sclerosis(Demyelinating) J) Smooth ependymal enhancement of ventriculitis(infective) K) Bilateral lentiform nucleus enhancement in metabolic encephalopathy L) Conglomerated tuberculomas showing smooth thin ring enhancement of infective etiology

majority of the lesions in our study were infective (56\%) and they showed smooth thin ring enhancement (26\%) and Leptomeningeal (22\%) enhancement. Pachymeningeal enhancement (14\%, 19 patterns) was the next common enhancement pattern and with meningiomas contributing the most common cause. (57.89\%, 11 cases). A rare case of meningioma also showed thick irregular ring enhancement. Thick irregular ring enhancement (12\%, 16 instances) is typical of malignant intraparenchymal brain tumors. 11 of these 16 patterns $(68.75 \%)$ were seen in cases of malignant brain tumors which included anaplastic astrocytoma, anaplastic Oligodendroglioma and high and low grade gliomas. Thick irregular ring enhancement was also noted in 2 cases of metastatic brain tumor. Metastatic brain tumors typically depicted nodular cortical-subcortical enhancement pattern. Of the 8 instances of nodular cortical-subcortical enhancement, $7(87.5 \%)$ were seen in cases of metastatic lesions which are typically located at gray matter-white 
matter junction in cortical subcortical location. A history of primary tumor was usually evident and facilitated the diagnosis.

\section{DISCUSSION}

The analyses of results from our study shows that specific enhancement patterns can pint point a definitive diagnosis in most cases as different etiologies have different patterns on contrast enhanced MRI.

There are two types of extraaxial enhancement patterns. ${ }^{2}$ The first type pachymeningeal enhancement can be seen in post operative patients as well as in benign neoplasms like meningiomas producing the so called classical dural flair or dural tail sign. ${ }^{1,2,6,7}$ Meningiomas are the most common primary brain tumors. ${ }^{8}$ The dural tail is nothing but a curvilinear dural enhancement probably caused by a reactive process occurring as a result of vasocongestion and edema. The dural capillaries lack a blood brain barrier resulting in contrast retention within dura mater and dural flair sign. ${ }^{1,2,9}$ Nevertheless, dural tail should always be examined at surgery so as to not leave any residual tumor tissue behind. Pachymeningeal enhancement can also be seen in intracranial hypotension, other granulomatous diseases like fungal infections, and secondary metastases to meninges. ${ }^{1,2}$ Leptomeningeal enhancement is seen in sulci and subarachnoid cisterns i.e pia-arachnoid enhancement. It is primarily due to break down of blood brain barrier without angiogenesis as is classical of meningeal infections. Bacterial and viral meningitis cause thin and linear type of piaarachnoid enhancement whereas thicker, lumpy or nodular enhancement is seen in fungal infections. ${ }^{1,10,11}$

Viral infections also produce another type of intraaxial enhancement called as superficial gyral enhancement. Vasodilatation after reperfusion of ischemic brain, the vasodilatation phase of migraine headache, posterior reversible encephalopathy syndrome (PRES), and vasodilatation with seizures result in abrupt onset of symptoms and serpentine gyral enhancement affecting single vascular territory. ${ }^{12-14}$ Infective or inflammatory causes of gyral enhancement affect multiple vascular territories and usually have a more indolent onset and nonspecific symptoms. Also, PRES lesions are seen in posterior circulation. ${ }^{1,2}$

Nodular cortical and subcortical enhancement pattern has a narrower differential diagnosis of metastasis or septic emboli. These lesions are classically seen as small $(<2 \mathrm{~cm})$ well circumscribed lesions at Gray-white matter junction which is the region of vessel branching and tapering wherein filtration of intravascular particulate materials occur and hence the type of enhancement in these cases.

If enhancing lesions are detected in deeper white matter, deep gray nuclei(basal ganglia, thalamus) and in periventricular locations, metabolic encephalopathies or toxoplasmosis or primary CNS lymphoma should be considered., ${ }^{1,2}$ Deep ring enhancing lesions can have several specific patterns. Smooth thin ring enhancement is most commonly observed with cerebral abscess(bacterial, tuberculous, parasitic etc). Tuberculomas may show conglomeration and a more thicker wall. ${ }^{15}$

Malignant CNS neoplasms like necrotic high grade gliomas show thick irregular ring enhancement. ${ }^{16-21}$ The ring has thicker medial wall $(>10 \mathrm{~mm})$ towards cortex, shaggy inner margin. Fluid secreting low grade primary neoplasms like pilocystic astrocytomas anad hemangioblastomas show enhancement limited to the mural nodule ${ }^{22}$, whereas some may demonstrate a nodule with partial rim enhancement (a variant of the open ring sign). Demyelinating lesions most commonly multiple sclerosis cause perivenular inflammation during active phase resulting in a thin incomplete ring enhancement with the lesions oriented perpendicularly to the margins of lateral ventricles. ${ }^{23,24,25}$ Periventricular enhancement can be smooth and thin $(<2 \mathrm{~mm})$ linear along the margins of ventricles in infectious ependymitis. Thick irregular shaggy(Lamb's wool pattern) periventricular enhancement can be seen in primary CNS lymphomas., ${ }^{1,2}$

A diagnostic approach to differential diagnosis based on enhancement patterns has been tabulated in table 1 .

A limitation that spurs out from comparison of enhancement patterns of infective and neoplastic etiologies (figure 2) clearly specifies that Leptomeningeal and smooth thin ring enhancement favours an infective etiology whereas thick irregular and pachymeningeal enhancement is more diagnostic of neoplastic etiology. However, as the above graph suggests, infective lesions may sometimes mimic neoplasms giving a thick irregular ring enhancement and neoplasms particularly metastases can result in a deceptive looking ring enhancement(cortical-subcortical).

Long standing infective lesions also sometimes result in a thick irregular pattern of enhancement typical of malignant neoplasms and hence can appear as tumor mimics. Careful clinical correlation with use of MR spectroscopy as and when required is needed to arrive at a clear diagnosis. Immediate post contrast imaging was done which underestimated contrast enhancement in some lesions which showed delayed enhancement. Only Post contrast fat saturated T1Weighted images were assessed with no availability of precontrast fat saturated $\mathrm{T} 1$ weighted images and thus baseline comparisons were difficult in some cases. Infective lesions like tuberculous meningitis showed some $\mathrm{T} 1$ post contrast enhancement in leptomeninges which could be negated using FLAIR post contrast images which were not evaluated in our study. Many lesions did not follow the enhancement patterns elucidated by smirniotopoulos et al 2007, and hence were not classified as per our enhancement patterns. Hence, we propose a study including $\mathrm{T} 1$ fat saturated precontrast images and post contrast FLAIR imaging, including a delayed T1 post contrast image in a study on a much larger population to elucidate more detailed and specific enhancement patterns. Also, some lesions did not conform to the delineated enhancement patterns and hence need specific studies on a larger sample to elucidate and classify such enhancement patterns to facilitate the imaging diagnosis.

\section{CONCLUSION}

Every etiology has its peculiar enhacement pattern (figure 3). A simple recognition of the type of enhancement pattern produced by brain lesions can easily spot the diagnosis and accelerate diagnostic work flow. Classifying an enhancement pattern of the brain lesion helps in easy grouping of lesions 
into their respective etiologies which can be narrowed down with the help of other MR sequences and a little clinical detail. This also further enables the clinician to institute prompt treatment and avoid unnecessary invasive procedures to establish etiology. However, enhancement pattern can not serve as a lone warrior to diagnosing a brain lesion in all cases and hence, a collaborative effort of clinical and imaging features, as always, is deemed necessary.

\section{REFERENCES}

1. Smirniotopoulos JG, Frances M. Murphy, Elizabeth J. Rushing, John H. Rees, Jason W. Schroeder. Patterns of contrast enhancement in the Brain and Meninges. Radiographics 2007; 27(1):525-551.

2. N. Arcalis, S. Medrano, D. Garcia, M. Cuadrado, A. Conejero Olesti, J. M. Ruiz restrepo, X. Pruna, C. Blancas, S. Carbo; Granollers/ES, Barcelona/ ES: Patterns of contrast enhancement in the brain and meninges. A Pictorial Review: http://dx.doi. org/10.1594/ecr2011/C-0716.

3. Marco Essig, Marc-Andre Weber, Hendrik von TenggKobligk, Michael V.knopp, William T.C. Yuh, Frederik L. Giesel. Contrast enhanced Magnetic resonance imaging of central nervous system tumors, Top Magn Reson Imaging 2006; 17(5):89-106.

4. Ji-Young Jeen et al. Effect of imaging time in MRI detection of intracerebral metastasis using single dose gadobutrol. Korean J Radiol 2014; 15(1). 145-150.

5. M. Essig, N. Anzalone, S.E. Combs, A. Do" rfler, S.K. Lee P. Picozzi, a` Rovira M. Weller M. Law. MR Imaging of Neoplastic Central Nervous System Lesions: Review and Recommendations for Current Practice. Am J Neuroradiol 2012; 33(2):803-17.

6. Burke JW, Podrasky AE, Bradley WG Jr. Meninges: benign postoperative enhancement on MR images. Radiology 1990; 174(6):99-102.

7. Mittl RL Jr, Yousem DM. Frequency of unexplained meningeal enhancement in the brain after lumbar puncture. Am J Neuroradiol 1994; 15(4):633-638.

8. Buetow MP, Buetow PC, Smirniotopoulos JG. Typical, atypical, and misleading features in meningioma. RadioGraphics 1991; 11(1):1087- 1106.

9. Nagele T, Petersen D, Klose U, Grodd W, Opitz H, Voigt K. The dural tail adjacent to meningiomas studied by dynamic contrast-enhanced MRI: a comparison with histopathology. Neuroradiology 1994; 36(3):303-307.

10. Spellerberg B, Prasad S, Cabellos C, Burroughs M, Cahill P, Tuomanen E. Penetration of the blood-brain barrier: enhancement of drug delivery and imaging by bacterial glycopeptides. J Exp Med 1995; 182(5):10371043.

11. Sage MR, Wilson AJ, Scroop R. Contrast media and the brain: the basis of CT and MR imaging enhancement. Neuroimaging Clin N Am 1998; 8(2):695-707.

12. Schaefer PW. Diffusion-weighted imaging as a problem-solving tool in the evaluation of patients with acute stroke like syndromes. Top Magn Reson Imaging 2000; 11(4):300-309.

13. Provenzale JM, Petrella JR, Cruz LC Jr, Wong JC, Engelter S, Barboriak DP. Quantitative assessment of diffusion abnormalities in posterior reversible encephalopathy syndrome. Am J Neuroradiol 2001;22(6):1455-1461.

14. Silverstein AM, Alexander JA. Acute postictal cerebral imaging. Am J Neuroradiol 1998;19(2): 1485-1488.

15. Mohindra S, Savardekar A, Gupta R, Tripathi M, Rane $\mathrm{S}$. Tuberculous brain abscesses in immunocompetent patients: A decade long experience with nine patients. Neurol India 2016;64(5):66-74

16. KleihuesP, Louis DN, Scheithauer BW, et al. The WHO classification of tumors of the nervous system. J Neuropathol Exp Neurol 2002; 61(3): 215-225.

17. GoebellE, Paustenbach S, and Vaeterlein O, et al. Lowgrade and anaplastic gliomas: differences in architecture evaluated with diffusion-tensor MR imaging. Radiology 2006;239(1):217-222.

18. Law M, Yang S, Wang $\mathrm{H}$, et al. Glioma grading: sensitivity, specificity, and predictive values of perfusion MR imaging and proton MR spectroscopic imaging compared with conventional MR imaging. Am J Neuroradiol 2003;24(1):1989-1998.

19. StadlbauerA, Gruber S, Nimsky C, et al. Preoperative grading of gliomas by using metabolite quantification with high-spatial-resolution proton MR spectroscopic imaging. Radiology 2006; 238(3): 958-969.

20. MillsSJ, Patankar TA, Haroon HA, Baleriaux D, Swindell R, Jackson A. Do cerebral blood volume and contrast transfer coefficient predict prognosis in human glioma? Am J Neuroradiol 2006;27(1): 853-858.

21. MullinsME, Barest GD, Schaefer PW, Hochberg FH, Gonzalez RG, Lev MH. Radiation necrosis versus glioma recurrence: conventional MR imaging clues to diagnosis. Am J Neuroradiol 2005; 26(5): 1967-1972.

22. Ho VB, Smirniotopoulos JG, Murphy FM, Rushing EJ. Radiologic-pathologic correlation: hemangioblastoma. Am J Neuroradiol 1992;13(3):1343-1352.

23. Schwartz KM, Erickson BJ, Lucchinetti C. Pattern of T2 hypointensity associated with ring-enhancing brain lesions can help to differentiate pathology. Neuroradiology 2006; 48(6):143-149.

24. Masdeu JC, Moreira J, Trasi S, Visintainer P, Cavaliere $\mathrm{R}$, Grundman $\mathrm{M}$. The open ring: a new imaging sign in demyelinating disease. J Neuroimaging 1996; 6(3):104107.

25. Masdeu JC, Quinto C, Olivera C, Tenner M, Leslie D, Visintainer P. Open-ring imaging sign: highly specific for atypical brain demyelination. Neurology 2000; 54(5):1427-1433.

\section{Source of Support: Nil; Conflict of Interest: None}

Submitted: 05-01-2020; Accepted: 01-02-2020; Published online: 29-02-2020 